\title{
An Evolution Process Model for the Internet Topology*
}

\author{
Sangjoon Park ${ }^{1}$, Insook $\mathrm{Cho}^{2}$, and Byunggi Kim ${ }^{2}$ \\ ${ }^{1}$ Information \& Media Technology Institute, Soongsil University \\ ${ }^{2}$ School of Computing, Soongsil University \\ $\{$ lub, bgkim\}@archi.ssu.ac.kr
}

\begin{abstract}
Instead of actual experiments to network protocols, network simulators are useful to analyze these network protocols for lower analysis cost. The Internet topology is dynamically evolving and growing, and then shows changing characteristics based on time flow. Studies of Internet topology have been motivated by the demands for analysis and simulation to the modeling of real networks. Hence, to develop the Internet simulator, proper characteristics to Internet topology should be studied. In this paper, we propose topology models to the Internet topology showing node addition and deletion.
\end{abstract}

\section{Introduction}

Real experiments to large scale network are ideal to correctly analyze performance evaluations to researched network protocols. However, it is very difficult since researched protocols cannot be directly tested in real networks, and the evaluation cost based on real experiments is very high. Hence, the modeling and the simulation to the large scale network such like Internet are useful to assess researched protocol performances. Advantages of the modeling and the simulation for such network structure are as follows:

-Evaluation cost: Generally, the experimental evaluation to the actual network is very difficult and expensive. However, by using a simulator to the source network system, it can spend much lower evaluation cost compared with actual experiments.

-Flexibility: The experimental implementation has the limitation of application flexibility caused by environment problems when an additional scheme is newly adapted to the source network systems. In simulation environments, the addition of a new scheme is much easier than it on the experimental environments.

-Applicability: Network simulators developed in the modeling can be used to analyze variety network mechanisms.

The Internet was developed for military and research-oriented prototypes, but it has grown into very large scale network that connects many computers around the world without any centralized control or administration. Hence, to implement simulations related to network protocols on the Internet, it is important to study the properties of and policies of the Internet (e.g., network scale, connection types between different

* This work was supported by the Soongsil University research fund. 
networks and network evolution model). Therefore, most researches to the Internet focus topology models that try to represent the characteristics of the real Internet topology accurately [1] [2] [7]. In this paper, we propose an Internet topology model that has the functionalities of adding and deleting nodes and processing the isolated nodes to accurately apply the changes in the real Internet. We consider a node change model for showing characteristics of Internet evolution.

\section{Internet Topology Property}

\subsection{Evolution Model}

There are power-laws among the several properties that characterize the Internet topologies [5], [6], [9]. At present, the well-known key power-laws are the following: First, the power-law relationship between the degree of a node $\left(d_{v}\right)$ and the rank of a node $\left(r_{v}\right)$ is presented by

$$
d_{v} \propto r_{v}{ }^{R}
$$

where $v$ is a node in the Internet, and $R$ is the power of a constant (rank exponent).

Secondly, a power-law appears between an degree $(d)$ and the frequency $\left(f_{d}\right)$ as follows:

$$
f_{d} \propto d^{o}
$$

where $O$ is the degree exponent..

Thirdly, a power-law is the relationship between a calculated eigen value $\left(\lambda_{i}\right)$ and a rank $(i)$ as follows:

$$
\lambda_{i} \propto i^{\varepsilon}
$$

where $\varepsilon$ is the eigen exponent.

The rank is arranged in ascending order by the eigen value using a close matrix. If all nodes are connected, it is represented by 1 , and if not connected, it is expressed by 0 .

\subsection{Topology Model}

Waxman model is the distance-based connection that links of nodes are added to a network considering the distance between nodes arranged in random positions on $2 \mathrm{D}$ grid [10]. The rate of generating link between the two nodes $u$ and $v$ is different depending on the distance of the node from the node, and it is determined using the connection rate as follows. Waxman model is usually used to create a random network.

$$
P(u, v)=\alpha e^{-\frac{d}{\beta L}}
$$

where $d$ is euclidean distance from node $u$ to $v, L$ is the maximum distance between two nodes in a graph, and $0 \leq \alpha, \beta \leq 1$.

Barabasi-Albert model is the degree-based connection method that a characteristic of the Internet evolution shows a power-law distribution by providing incremental growth and preferential connectivity to the degree frequency [3][4]. 


$$
P(j)=\frac{d_{j}}{\sum_{k \in V} d_{k}}
$$

where $d_{j}$ is the degree of the node $(j)$ and $V$ is the collection of all nodes connected in the network. $\sum_{k \in V} d_{k}$ is the degree sum of all existing nodes.

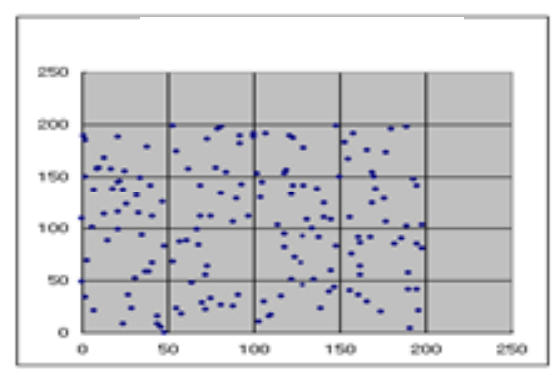

Fig. 1. Random topology (Arrangement of nodes without links)

\section{Internet Topology Model}

In this paper, we design an Internet topology model showing changeable network configuration. In the time flow, network nodes can be deleted or added to the growing Internet topology.

\subsection{Method to Make a Topology in the Beginning}

Firstly, to present the initial Internet topology, we use the node information (1999 year-network information) to the Korea Internet network. The node generation pattern is that the initial node is generated and then will be arranged in the network. Furthermore, after creating a node in small-sized topology, the arrangement to the node uses a random method. A link connection between two nodes uses the node connection rate [equation (5)] in Waxman model and the degree-based connection method [equation (6)]. Fig. 1 shows the beginning topology created using a random method. The AS number is 149 in 1999.

\subsection{Node Addition}

After initial topology generation, if a new node is created and is connected to another node, the node addition method is same as above mentioned subsection 3.1: Firstly, the node connectivity rate considering Euclidean distance between two nodes and secondly degree-based connection method using the connectivity rate of the Barabasi-Albert model. In Barabasi-Albert model, a higher degree node presents higher connectivity rate in the existing network [3] [10]. 


\subsection{Node Deletion}

Current Internet models only consider the network growing modeling. However, note that a network node can be deleted from social or technical problems. Hence, though the Internet is continuously growing, each network node has its life cycle so that it can be died. In this paper, we derive two node deletion scenarios based on equation (5) and (6). First scenario is based on the distance between two nodes as follows.

$$
P_{i}=\frac{l_{i}}{\sum l_{k}}
$$

where $l_{i}$ is the distance value of a node $i$, and $\sum l_{k}$ is the distance sum of all nodes.

In distance-based scheme, if a node $(i)$ is far away from a core node and has the maximum distance value $l_{i}$, the rate $P_{i}$ to $i$ is the maximum value. Hence, the node $i$ having the maximum rate value will be deleted. For the second method, it is the case that considers the degree and the distance simultaneously. Hence, even though a node has the maximum distance value, if the degree of node is high, the node can alive. If $\left\{I_{0}, I_{1}, I_{2}, \cdots, I_{n}\right\}$ is the distance set of all nodes from a core node, $\left\{d_{0}, d_{1}, d_{2} \cdots, d_{n}\right\}$ is the degree set of all nodes, and the total number of node is $m$, the rate $P_{i}$ is given by

$$
P_{i}=\psi \frac{l_{i}}{\sum_{i \in V} l_{i} / m}-\delta \frac{d_{i}}{\sum_{i \in V} d_{i} / m}
$$

where $\psi$ and $\delta$ are the shape parameters, $\sum_{i \in V} I_{i} / m$ and $\sum_{i \in V} d_{i} / m$ are the average values, and $V=\{0,1,2, \cdots, n\}$ is the set of all nodes.

Here, we use the average value for each node to make the same effects about the degree and the distance.

\subsection{Processing an Isolated Node}

After deleting a core node or a node having high degree, the process to isolated neighbor nodes (with zero degree) is required. Hence, it needs to create a new link and

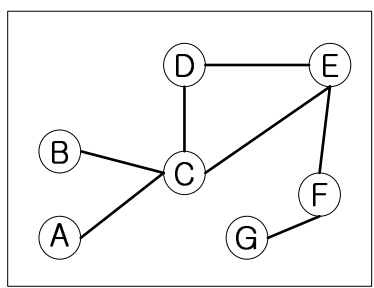

(a)

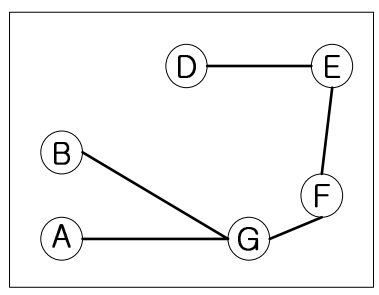

(b)

Fig. 2. Processing an isolated node: (a) Before node deletion and (b) After node deletion 
connect to another node. As an example, Fig. 2 shows the node isolation process. In Fig. 2(a), the node $(C)$ has 4 degrees. If the node $(C)$ is deleted, node $(A)$ and node $(B)$ are isolated. Hence, node $(A)$ and node $(B)$ should be connected another node [here, node $(G)$ ]. To create new links, it uses a method similar to that of adding a link when a new node is created: Waxman's node connectivity rate [equation (5)] and the degree-based connection method [equation (6)].

\section{Performance Evaluation}

We evaluate the correlation between each topology model and the power-law by using three power-law equations (in Section 2.1) [8]. To evaluate the proposed model, we created the network topology in two ways. Table 1 show the current network models without the node deletion function, and Table 2 presents the proposed models that includes the node deletion function and the isolated node process. In the distance-based method, Waxman parameter $\alpha$ and $\beta$ are 0.6 and 0.4 , respectively [see equation (5)].

Table 1. Topology model without a deletion function

\begin{tabular}{|c||c|c|}
\hline \multicolumn{1}{|c||}{\multirow{2}{*}{$\begin{array}{c}\text { Model } \\
\text { name }\end{array}$}} & \multicolumn{2}{c|}{ Node addition } \\
\cline { 2 - 3 } & Node creation & Link connection \\
\hline \hline A1 & Incremental method & Distance-based method \\
\hline A2 & Incremental method & Degree-based method \\
\hline
\end{tabular}

Table 2. Topology model with a deletion function

\begin{tabular}{|c|c|c|c|c|}
\hline \multirow{2}{*}{$\begin{array}{c}\text { Model } \\
\text { name }\end{array}$} & \multicolumn{2}{|c|}{ Node addition } & Node deletion & Processing to isolated nodes \\
\cline { 2 - 3 } B1 & $\begin{array}{c}\text { Node Creation } \\
\text { Incremental } \\
\text { method }\end{array}$ & $\begin{array}{c}\text { Link connection } \\
\text { Distance-based } \\
\text { method }\end{array}$ & Distance-based method & Distance-based method \\
\hline B2 & $\begin{array}{c}\text { Incremental } \\
\text { method }\end{array}$ & $\begin{array}{c}\text { Degree-based } \\
\text { method }\end{array}$ & Distance-based method & Distance-based method \\
\hline B3 & $\begin{array}{c}\text { Incremental } \\
\text { method }\end{array}$ & $\begin{array}{c}\text { Degree-based } \\
\text { method }\end{array}$ & Distance-based method & Degree-based method \\
\hline B4 & $\begin{array}{c}\text { Incremental } \\
\text { method }\end{array}$ & $\begin{array}{c}\text { Degree-based } \\
\text { method }\end{array}$ & $\begin{array}{c}\text { Degree and distance-based } \\
\text { method }\end{array}$ & Distance-based method \\
\hline B5 & $\begin{array}{c}\text { Incremental } \\
\text { method }\end{array}$ & $\begin{array}{c}\text { Degree-based } \\
\text { method }\end{array}$ & $\begin{array}{c}\text { Degree and distance-based } \\
\text { method }\end{array}$ & Degree-based method \\
\hline
\end{tabular}

In this paper, we used the status of the number of national AS announced by the Korea Network Information Center (KRNIC) [11]. The yearly status of the number of AS to Korea and the world is shown in Table 3. The actually used data in this simulation is from January 1999 to May 2003. 
Table 3. AS number from 1999 to 2003

\begin{tabular}{|c||c|c|c|c|}
\hline \multirow{2}{*}{ Year } & \multicolumn{2}{|c|}{ Korea } & \multicolumn{2}{c|}{ World } \\
\cline { 2 - 5 } & AS & Growth rate & AS & Growth rate \\
\hline 1999 & 149 & 1.00 & 11,232 & 1.00 \\
\hline 2000 & 300 & 2.01 & 15,710 & 1.39 \\
\hline 2001 & 391 & 2.62 & 20,128 & 1.79 \\
\hline 2002 & 433 & 2.90 & 23,822 & 2.12 \\
\hline 2003 & 494 & 3.31 & 24,827 & 2.21 \\
\hline
\end{tabular}

\subsection{Evaluation Results}

Unfortunately, we can show evaluation results to only the model A2 and the model B5 because of the limited paper space. Fig. 3 and Fig. 4 show graphs that the network topology grows from 149 AS nodes to AS 494 nodes. Hence, to the network growth, the power-law is examined by the growth characteristic of each topology, and results are plotted on the Log-log scale. Fig. 3 is a result using the model (A2) without a node deletion function.

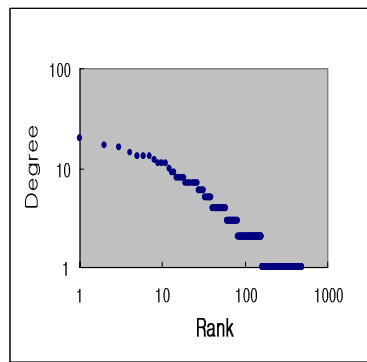

(a) CC: -0.95

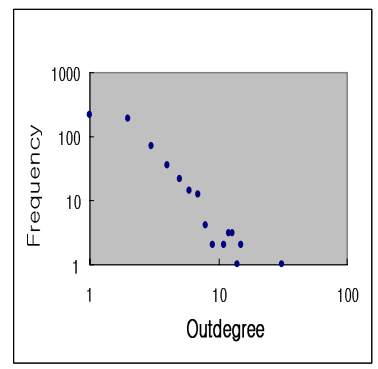

(b) CC: -0.95

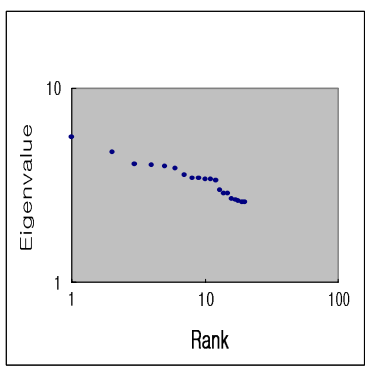

(c) CC: -0.97

Fig. 3. The network model (A2) : (a) Rank versus degree (b) Degree versus frequency and (c) Rank versus eigenvalue

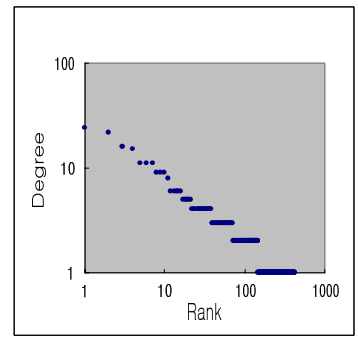

(a) CC: -0.96

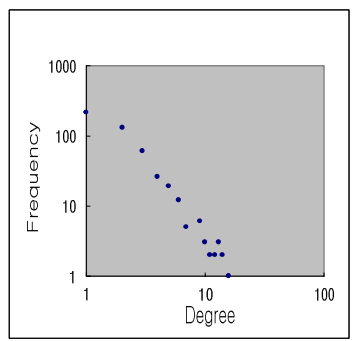

(b) CC: -0.98

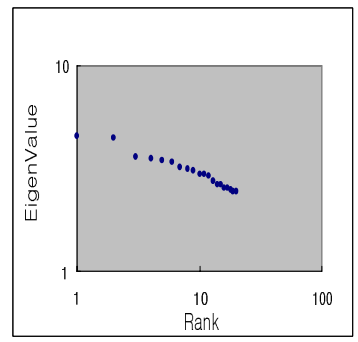

(C) CC: -0.98

Fig. 4. The network model (B5) : (a) Rank versus degree (b) Degree versus frequency (c) Rank versus eigenvalue 


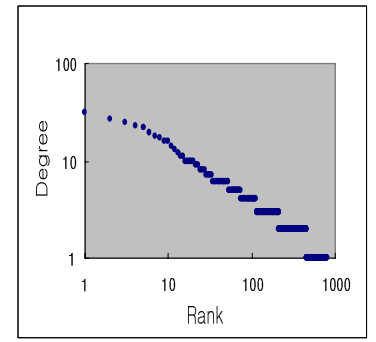

(a) CC: -0.96

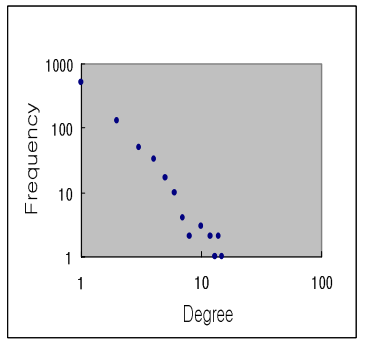

(b) CC: -0.98

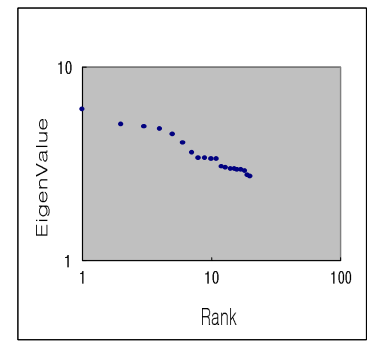

(C) CC: -0.98

Fig. 5. The predicted network model (B5) after 3 years: (a) Rank versus degree (b) Degree versus frequency and (c) Rank versus eigenvalue

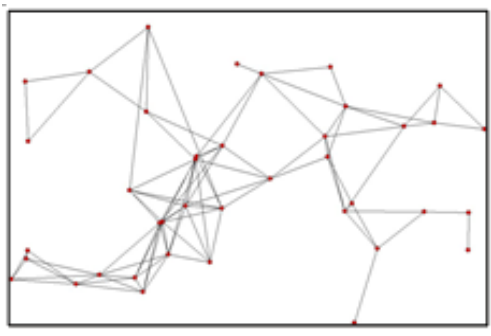

(a)

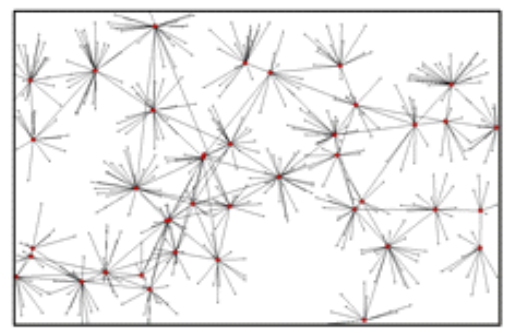

(b)

Fig. 6. A network topology example based on the model (B5): (a) core nodes (b) core nodes with leaf nodes

In Fig. 3, three sub-graphs [(a), (b) and (c)] show that each result presents the power-law property because A2 uses a node addition using a gradual increase method and a link creation using the degree-based method. Each CC is $-0.95,-0.95,-0.97$. Here, $\mathrm{CC}$ means the correlation coefficients between the network model and the power-law property. Fig. 4 shows the result of using the model (B5) including a node deletion function and the isolated node process. In Fig. 4 , each CC is $-0.96,-0.98$ and -0.98 . The correlation coefficient for the model (B5) show better results of 0.01, 0.03, 0.01 than the ones for the A2 model. Fig. 5 shows the prediction results to the model (B5) after 3 years. We determine the future node number by calculating the average value to all of growth rates in Table 3. After 3 years, the correlation coefficient of B5 is $-0.96,-0.98$, -0.98 . The correlation coefficient of the model (B5) shows better results than that of the model (A2) with $0.01,0.03$ and 0.02. Fig. 6 shows an example of the network topology by the model (B5). The total number of AS node is 490. In Fig. 6(a), the core nodes are firstly generated, and the leaf nodes having the low degree are secondly generated and connected to the core nodes in Fig. 6(b). The proposed model in this paper deals not only with the addition of nodes but it also applies to the node deletion. Hence, we can show more elaborate network model for the Internet evolution. 


\section{Conclusions}

In this paper, we propose an Internet topology model reflects the status of changing topologies caused by the creation and deletion of nodes. The model that we have proposed with the function of node deletion reflects the real Internet topology modeling better compared with current models that simply adds nodes. Furthermore, it shows that the proposed model (B5) has better result values than the model (A2) for predicting the Internet topology in the future. Therefore, the proposed model can better satisfy the power-law that is the characteristic of the Internet compared with current models without the deleting function. We hope the proposed network model that can apply to the topology generator to implement the Internet protocol simulation.

\section{References}

1. Zegura, E.W., Calvert, K. L. and Donahoo, M. J., "A Quantitative Comparison of Graph-based Model for Inter-networks,” IEEE/ACM Transactions, vol. 5, no. 6, pp.770-783, December 1996.

2. Albert, K. L., Doar, M. B. and Zegura, E. W., "Modeling Internet Topology," IEEE Communications Magazine, Vol. 35, No. 6, pp. 160-163, June 1997.

3. Barabasi, A. L. and Albert, R., "Emergence of Scaling in Random Network," Science, Vol. 286, pp. 509-512, October 1999.

4. Barabasi, A. L., Albert, R. and Jeong, Hawoong, "Scale-free Characteristics of Random Networks: the Topology of the WWW," Physica A, Vol. 28, No. 1, pp. 69-77, 2000.

5. Chen, Q., Chang, H., Govindan, R., Jamin, S., Shenker, S. and Willinger, W., "The Origin of Power Laws in Internet Topologies Revisited," In Proceeedings of IEEE INFOCOM 2002, Vol. 2, pp. 23-27, June 2002.

6. Faloutsos, M., Faloutsos, P. and Faloutso, C., "On Power-LawRelationships of the Internet Topology," In Proceedings of ACM Computer Communication Review, pp. 251-261, September 1999.

7. Jin, C., Chen, Q. and Jamin, S., "Inet: Internet Topology Generator," Technical Report CSE-TR-433-00, University of Michigan at Ann Arbor, 2000.

8. Medina, A., Lakhina, A., Matta, I. and Byers, J., "BRITE: An Approach to Universal Topology Generation," In Proceeding of MASCOTS 2001, pp. 346-353, August 2001.

9. Medina, A., Matta, I. and Byers, J., "On the Origin of Power Laws in Internet Topologies," Computer Communication Review, Vol. 30, No. 2, pp. 18-28, April 2000.

10. Waxman, B. M., "Routing of Multipoint Connections," IEEE Journal on Selected Areas in Communications, Vol. 6, No. 9, pp. 1617-1622, December 1998.

11. http://www.krnic.or.kr. 